\title{
Anthropologies parallèles : description d'une procédure d'Enquête Anthropologique Rapide (EAR)
}

Jacky Bouju

\section{(2) OpenEdition Journals}

Édition électronique

URL : https://journals.openedition.org/apad/1591

DOI : 10.4000/apad.1591

ISSN : 1950-6929

Éditeur

LIT Verlag

\section{Édition imprimée}

Date de publication : 1 juin 1995

\section{Référence électronique}

Jacky Bouju, «Anthropologies parallèles : description d'une procédure d'Enquête Anthropologique Rapide (EAR) », Bulletin de l'APAD [En ligne], 9 | 1995, mis en ligne le 26 juillet 2007, consulté le 21 septembre 2021. URL : http://journals.openedition.org/apad/1591 ; DOI : https://doi.org/10.4000/ apad. 1591

Ce document a été généré automatiquement le 21 septembre 2021.

Bulletin de I'APAD 


\section{Anthropologies parallèles : description d'une procédure d'Enquête Anthropologique Rapide (EAR)}

Jacky Bouju

1 Dans le champ du développement, l'anthropologie a été longtemps tenue à l'écart des études de "faisabilité" - qui prennent place en amont de l'intervention (consistant, généralement, en une description du milieu social local) - ou des "évaluations" qui tentent de comprendre, plus que mesurer, l'impact d'une intervention. Cette mise à l'écart s'est réalisée pour le plus grand profit d'autres disciplines comme la socio-économie ou la géographie humaine qui ont allègrement "piraté" l'anthropologie (en termes d'emprunts de méthodes et techniques d'enquête, mais aussi de concepts et de notions appartenant à son jargon théorique) avec les résultats que l'on sait. Aujourd'hui, la tendance semble s'inverser et, de plus en plus souvent, c'est à l'anthropologie qu'on réclame une expertise sociale. L'anthropologue ne peut que se réjouir de ce recours à condition, toutefois, qu'il exprime une véritable reconnaissance disciplinaire, ce qui est loin d'être sûr! Mais cette discussion déborde largement le propos que je veux tenir ici.

2 L'impression dominante qui se dégage de mon expérience en matière de E.AR. (Enquête Anthropologique Rapide pour traduire approximativement R.A.P. Rapid Anthropological Procedure) ${ }^{1}$ est que les demandeurs d'anthropologie (bailleurs de fonds, techniciens des autres disciplines, chefs d'entreprises ou de projets, responsables de programmes de développement, etc.) méconnaissent en général notre discipline et, à cause de cela, la créditent de prouesses explicatives qu'elle serait bien en peine de fournir (c'est ce qui, précisément, est inquiétant dans l'attrait qu'elle semble exercer depuis peu) mais qu'en tout état de cause, ils ont renoncé à obtenir de la part des autres sciences sociales, qui ont perdu, à leur yeux, une bonne partie de leur crédibilité heuristique. 
3 Je ne m'attacherai pas à ressasser les différences et les préjugés - en termes de points de vue, d'approches, ou de conceptions - qui existent entre les anthropologues et les professionnels du développement (elles ont déjà été soulignées ailleurs: Olivier de Sardan, 1990; Bouju, 1991; Desclaux, 1992, etc.). Je voudrai tenter une sorte d'auto-évaluation ${ }^{2}$, qui sera donc partiale et partielle, d'une enquête anthropologique rapide (EAR) afin d'essayer, à la faveur du laps de temps écoulé (mise au point en 1988 et application en 1989), d'en préciser la pertinence et les limites applicatives. Il semblerait, en effet, que les méthodes EAR soient en passe de devenir un enjeu important (Desclaux, A. 1992 ; Bierschenk, T. \& Olivier de Sardan, J.-P. 1994 ; Gruénais, M.-E. 1994 ; Vidal, L. 1994) en tant que supports possibles d'un renouveau du dialogue entre anthropologues et professionnels du développement ${ }^{3}$. Par rapport à cet enjeu, je me rangerai plutôt dans le courant des anthropologues qui pensent que "les guides méthodologiques n'offrent qu'une présentation formelle de méthodes déjà utilisées jusque-là" (Desclaux, 1992 : 305). Mais je pense aussi que la formalisation présente un certain intérêt, en particulier au plan pédagogique. L'EAR que je propose est du type "à problématique minimum incorporée" ( $c f$ Bierschenk,T. \& Olivier de Sardan, J.-P.,1994 : 35) et sa présentation, ici, vise à fournir des éléments supplémentaires qui puissent contribuer à nourrir le débat en cours sur les EAR.

Historique de la démarche

4 À l'origine de la méthode on trouve ce qu'il convient d'appeler une demande sociale. Cette demande qui m'était adressée, émanait d'un agronome du programme de développement rural U.P.1-Zorgo (province du Ganzourgou, Burkina Faso) qui formulait ainsi le besoin d'une connaissance anthropologique susceptible d'orienter l'action du projet :

Expliquer la situation de "blocage" que rencontre la mise en place des Contrats

Villageois de Développement (C.V.D.) avec les communes et les villages de la

province du Ganzourgou et proposer au projet des procédures pour sa résolution.

Les agents du projet constataient une situation d'indifférence - et parfois même de refus catégorique - de la part des "destinataires paysans" pour participer à la mise en place des C.V.D. Et pourtant, la conception de ces contrats semblait véritablement novatrice puisqu'elle engageait les cadres locaux du projet à négocier avec les autorités villageoises et à considérer les destinataires paysans comme des "partenaires responsables" capables de "négocier" et de "s'engager" dans un "rapport contractuel". La modernité qui ressortait d'une telle conception de l'interaction développeur/ développé ainsi que les réactions négatives du milieu paysan excitèrent ma curiosité et je voulus en savoir plus. Fort de mon acceptation, il restait quand même à mon collègue agronome à convaincre sa propre hiérarchie qui, après avoir longuement hésité ${ }^{4}$, a finalement pris la décision d'engager une étude "socio-anthropologique" ${ }^{5}$ pour comprendre ce qui "bloquait" la négociation du côté paysan.

La problématisation de la demande

6 Cependant, bien avant d'obtenir l'accord de principe des autorités du projet et des bailleurs de fonds, j'avais du proposer les termes de référence qui décrivaient l'intervention d'anthropologie appliquée. La notion très technique de "termes de référence" recouvre tout un travail de problématisation de la demande initiale qui consiste - en se posant les bonnes questions et en formulant les hypothèses adéquates à effectuer une interprétation anthropologique de cette demande. L'interprétation anthropologique est une traduction c'est-à-dire le fruit de plusieurs reformulations 
consécutives à une série d'entretiens conduits avec différents agents et responsables du projet. D'emblée, mon objectif était d'essayer de comprendre non pas pourquoi les paysans refusaient une conception tout à fait novatrice du développement rural, mais bien plutôt ce que l'appellation "destinataire paysan" utilisée par le projet recouvrait comme réalité locale d'une part et comme représentation dans le jargon du projet d'autre part. Autrement dit, quels étaient les interlocuteurs locaux qui avaient été identifiés par le projet comme susceptibles de conclure avec lui un "Contrat de Développement Villageois". A la suite de quoi, je me trouvais en mesure de faire au moins deux constats :

7 1. Il apparaissait que le projet avait engagé ses activités dans les villages de la province sans disposer de données anthropologiques descriptives préliminaires sur le milieu socio-culturel d'intervention.

La zone à étudier comprenait les 7 départements de la province du Ganzourgou au Burkina Faso. Cette province totalisait environ 300 "villages". Cette région est caractérisée par la persistance de formes coutumières (c'est-à-dire "précoloniales" dans ce contexte) de tenure foncière qui sont multiples et complexes (Bouju, 1991). Ces modes de tenure - qui reposent sur des légitimités historico-culturelles qui n'ont ni le même âge, ni les mêmes fondements - délimitent des espaces d'appropriation et des territorialités de natures différentes. Par ailleurs, chacun de ces modes d'appropriation sociale, symbolique, économique ou politique de l'espace a historiquement engendré ses propres institutions avec, localement, des niveaux spécifiques de décision.

8 2. Ensuite, et ceci est une conséquence directe du premier constat, les agents du projet ignoraient (mais j'ai appris, plus tard, qu'ils feignaient d'ignorer !) la complexité des réalités sociales paysannes et donc ne la prenaient pas en considération pour la conduite de leurs interventions locales.

Cette situation semblait témoigner d'une double méconnaissance dont on a pu observer les effets par ailleurs ${ }^{6}$. D'abord, les techniciens du projet ne s'interrogeaient pas le moins du monde sur les présupposés véhiculés par leur jargon technique. Aussi devinrent-ils mal à l'aise quand l'ethnologue mit en cause l'adéquation de ce jargon aux réalités socio-économiques locales.

10 Parmi les présupposés, un des plus communs et des mieux partagés est le stéréotype communautaire et consensuel ${ }^{7}$ (critiqué par Olivier de Sardan $1990: 31$ ) que l'on continue toujours d'appliquer au monde villageois. Je devais faire admettre par le projet que la tentative de résolution du problème posé passait, en partie, par le fait d'étudier la réalité sociale paysanne telle qu'elle était et non pas comme on croyait qu'elle fut.

Dans le monde rural du Ganzourgou, comme ailleurs, des individus, des groupes, des responsables coutumiers de quartiers détiennent une autorité, un savoir ou un pouvoir de dire et/ou de faire, que d'autres ne possèdent pas! En particulier, la chefferie administrative de village et les CDR étaient considérés par les villageois comme des médiateurs aux affaires extérieures tirant l'essentiel de leur légitimité du pouvoir politique central, c'est-à-dire sans grande autorité au niveau local. Cet état de fait, assez courant en Afrique de l'Ouest, n'empêchait pas les autorités administratives régionales, nationales et les projets de développement de considérer ces médiateurs comme seule autorité représentative ${ }^{8}$ au niveau local. Tout le problème est que s'ils représentaient effectivement le pouvoir central, ils ne représentaient pas le pouvoir local sur lequel ils n'avaient bien souvent aucune autorité.

Un autre exemple de ce problème est bien illustré par l'usage des notions de "village" et de "terroir" qui sont apparues comme centrales dans cette étude. Non 
seulement ces notions correspondaient à un découpage exogène ${ }^{9}$ inadéquat pour décrire et conceptualiser l'habitat mossi mais en plus, on présupposait une correspondance a priori entre un village et un terroir. En fait, le problème n'était pas d'utiliser des catégories pertinentes mais de trouver à tout prix des "interlocuteurs collectifs". Le niveau "village" du découpage des réalités locales "... recoupe ainsi paradoxalement les exigences (politiques) d'une commode administration des populations."(Olivier de Sardan, 1990).

Autocritique

11 La seconde forme de méconnaissance confirmait ce que j'avais déjà pu constater en d'autres circonstances, à savoir qu'on feignait d'ignorer ${ }^{10}$ les logiques, les intérêts et les stratégies des "destinataires paysans". Coutumier de cet état de chose, j'ai fait l'erreur de croire que mes interlocuteurs (les "décideurs" du projet) croyaient au discours technocratique qu'ils me tenaient. Tant et si bien que j'ai pris pour une ignorance des réalités paysannes locales ce qui n'était que de l'indifférence à ces mêmes réalités que, parfois même, on connaissait de l'intérieur.

En y réfléchissant ultérieurement, je ne pus me convaincre de la naïveté d'une telle méprise. Je pense aujourd'hui que, inconsciemment, j'ai "voulu croire" qu'il émanait des praticiens du développement une vraie demande de connaissance anthropologique appliquée. J'avais tellement souhaité que soit enfin reconnue l'utilité de la discipline dans ce domaine du développement qu'en cette affaire j'ai pris mon désir pour la réalité.

13 Ainsi, la Direction du projet m'avait bien dissimulé son manque de conviction sur l'utilité d'une étude anthropologique pouvant fournir des "clés explicatives" qui permettraient de débloquer les négociations en cours. Elle le fit d'autant mieux que la relance des négociations avec les paysans constituait véritablement pour elle un enjeu important. Cette méprise initiale sur la méconnaissance, apparente, du milieu paysan ne m'avait pas permis de voir en quoi l'acceptation d'engager une étude anthropologique des trames foncières avait constitué un enjeu de pouvoir intermédiaire, en forme de contrainte certes, mais qui permettait à la Direction de se trouver en position favorable pour emporter l'enjeu principal que représentait le refinancement quadriennal ${ }^{11}$ du projet par les bailleurs de fonds.

L'hypothèse de travail

14 La compilation des savoirs préalables permettait de formuler des hypothèses présentant un fort indice de plausibilité. Mais celles-ci devaient être contextualisées afin de produire des résultats précis susceptibles de répondre à la demande spécifique.

Le contexte général de l'intervention présentait les caractéristiques suivantes : la situation générale dans la province était celle d'une raréfaction des terres arables disponibles consécutive à l'augmentation des densités de population rurale, à la dégradation durable de la pluviométrie et des sols et à la généralisation des rapports marchands. Dans ce contexte, l'accès à de bonnes terres (les bas-fonds) est devenu un enjeu socio-économico-politique local extrêmement important pour les paysans qui revendiquent la prééminence de leurs droits fonciers. Or le recouvrement ou l'intersection en forme de trames des multiples modes d'appropriation du sol (situation identifiée au préalable) posait le problème de la reconnaissance des différents droits (renvoyant à différents types de légitimité) et de leur hiérarchisation interne. En fait. c'est tout le problème de la reconnaissance collective d'un ordre de prééminence entre les formes de droit foncier qui se pose aujourd'hui avec d'autant plus d'acuité qu'il était ignoré par la coutume ${ }^{12}$. Ce vide juridique s'explique par le contexte historique précolonial d'émergence de ces droits de contrôle et d'appropriation foncière: ceux qui n'étaient pas de même 
nature ou qui n'obéissaient au même principe ne pouvaient absolument pas être rangés selon un ordre unique de prééminence. L'approche par l'analyse des conflits ${ }^{13}$

Dans un tel contexte des situations de tensions et de conflits, émergent régulièrement entre personnes, familles, groupes d'intérêts et quartiers. Du point de vue anthropologique, les soit-disant "blocages" témoignent, avant tout, de la dynamique propre aux logiques des intérêts paysans et aux stratégies de la politique locale. Car l'ethnologue sait que le monde rural est fortement différencié, clivé sur divers plans (religieux, économique, politique, coutumier, sociaux etc.) et stratifié de multiples manières.

Chacun y va de sa stratégie propre vis-à-vis du projet et/ou de l'administration provinciale qui apparaissent (sans qu'ils en soient forcément clairement conscients) comme capables d'arbitrer et de décider de l'éminence des droits des uns et des autres et de la prééminence des uns par rapport aux autres. Il s'ensuit une situation de tension permanente traduisant des clivages internes et des divisions profondes qui se manifestent régulièrement par des conflits de groupes ou de quartiers au sein des "communautés villageoises" (sur lesquelles continuent de s'appuyer toutes les stratégies de la politique micro-locale).

Ces divisions et ces conflits internes non seulement interfèrent dans les interactions susceptibles de drainer des ressources extérieures mais le plus souvent ils se nouent ou éclatent à l'occasion de ces interactions. L'hypothèse fondamentale que je formulais était qu'il fallait chercher dans cette direction, c'est-à-dire localiser et identifier les conflits locaux dont l'analyse me paraissait susceptible d'éclairer les raisons du "blocage" des négociations autour des C.D.V. avec le projet provincial. L'analyse par les conflits présentait à mes yeux un autre avantage, économique celui-là : la certitude de gagner $d u$ temps (cf. Bierschenk \& Olivier de Sardan 1994 : 37) dans notre travail d'identification des causes de blocage. L'élaboration de la problématique s'achève ici avec la formulation des hypothèses de travail à partir desquelles on peut proposer des objectifs, des moyens et des techniques d'enquête.

La formulation de l'hypothèse explicative (cf. supra) permettait de subdiviser l'objectif général de l'étude en six objectifs intermédiaires :

1. Identifier les modalités locales d'appropriation, de contrôle et d'accès à la terre, au niveau micro-local, villageois et micro-régional de la province ;

2. Identifier et localiser les différentes autorités ou pouvoirs locaux détenteurs de droits de contrôle sur l'espace selon les modalités identifiées en 1 ;

3. Qualifier et déterminer leur aire d'influence dans l'espace et sur les groupes sociaux localisés ;

4. Cartographier les limites administratives des départements et des provinces, les limites coutumières des chefferies et les limites symboliques des maîtrises religieuses de la terre ;

5. Déterminer à quelles réalités conçues et/ou vécues par les paysans renvoyaient les notions de "terroir" et de "village" ;

6. Analyser les interférences ou les conflits produits par les recouvrements et les intersections des différentes trames foncières, historiques et administratives ainsi mises en évidence.

17 Méthodologiquement, la difficulté consiste avant tout à définir les niveaux de la recherche, ses différentes phases, les axes d'approche des problèmes et les techniques d'enquête de terrain en fonction des contraintes habituelles de durée d'étude, des financements consentis, des ressources humaines disponibles, de la coordination entre différents calendriers d'activités, et de bien d'autres petites choses encore. 
Le calendrier d'exécution a fait, comme il est de coutume, l'objet de négociations serrées entre l'ethnologue et la Direction du projet: l'accord est intervenu sur la base de 6 mois (24 semaines de janvier 1989 à juin 1989). Les activités ont été ainsi réparties :

1. Compilation et séminaire de préparation: mois (30 jours) où l'ethnologue compile les savoirs préalables pendant qu'il procède simultanément à la formation des enquêteurs (4) au traitement de la problématique spécifique à cette enquête ;

2. Enquête de terrain : 3 mois ( 90 jours)

2.1. Volet formation par l'enquête (10 jours) : encadrement de 10 étudiants en stage de terrain pour la licence de sociologie.

2.2. Enquête proprement dite : 4 enquêteurs permanents (étudiants en sociologie et en droit, niveau maîtrise). 2/7 départements couverts par enquêteur $=1,5$ mois d'enquête par département). Soit au total $=360$ jours d'enquête.

3. Exploitation et analyse des données: mois d'analyse des résultats: 30 jours / chercheur (2)

4. Réalisation du rapport final : mois pour la rédaction du rapport et la réalisation des cartes : 30 jours / chercheur (2)

La rapidité de l'E.A.R. : un problème pour la validation des données

Chacun sait que la méthode ethnographique (l'observation-participation) qui est utilisée, normalement, pour conduire une recherche anthropologique exige nécessairement un séjour de longue durée sur le terrain. Toute méthode EAR est donc intrinsèquement porteuse d'un sérieux problème méthodologique : celui posé par sa rapidité. Il convient, cependant, de remarquer que "La notion de 'rapidité' est très variable selon les enquêtes réalisées" (Desclaux 1992 : 302) et qu'elle peut recouvrir des périodes allant de 4 semaines (recueil simple des données) à 2 ans (pour le recueil et l'analyse des données).

Un certain nombre de "précautions" méthodologiques doivent donc être prises pour compenser la brièveté relative de l'enquête et améliorer la validation des informations obtenues dans un laps de temps que l'ethnologue trouvera toujours trop court.

Le savoir de l'ethnologue et ses conditions d'application

1. La première solution méthodologique que je propose à ce problème de validation pose que l'ethnologue doit nécessairement détenir au préalable, une très bonne connaissance du milieu. Or, une telle compétence ne peut être acquise que de deux manières :

- Soit la région concernée est le terrain de l'ethnologue. Cela signifie qu'il a effectué une recherche de longue durée (fondamentale ou autre) dans la zone concernée par l'intervention.

- Soit l'ethnologue aura vécu assez longtemps sur place pour acquérir un savoir propre (observation directe des réalités et participation quotidienne à la vie sociale locale) et pour compiler (par la maîtrise des sources existantes) les savoirs antérieurs accumulés sur le milieu social et la culture concernés par le projet.

21 Selon moi, ces deux points tracent la limite inférieure en-deçà de laquelle une Enquête Anthropologique Rapide ne peut être appliquée. C'est parce que l'étude ethnographique a été réalisée, dans la longue durée, avant et en dehors du cadre de l'expertise, que l'EAR (qui s'appuie sur la compilation ethnographique) est une méthode anthropologique complémentaire malgré la brièveté de son application. Ce que Bierschenk \& Olivier de Sardan (1994 : 35) disent d'ECRIS est parfaitement valable pour MAR: "C'est plutôt une méthode complémentaire du "terrain" classique, lequel reste indispensable et réclame une investigation individuelle relativement intensive et donc prolongée." 
22 2. En second lieu, et selon les moyens dont on peut disposer, on met en place un ensemble de procédures méthodologiques visant à garantir les résultats. Outre la compilation des savoirs préalables à partir des sources disponibles (cf. supra), je décidais de travailler en équipe et, à cette fin, dès l'acceptation des termes de référence par le projet, je m'adjoignis la collaboration d'un collègue anthropologue (chercheur senior). Nous assurâmes tous deux la direction et le suivi de l'enquête qui fut réalisée par quatre enquêteurs (étudiants en maîtrise de sociologie que nous formâmes à l'enquête). Enfin, un groupe de dix étudiants stagiaires (licence de sociologie) participa à l'enquête pendant dix jours. Ici, l'objectif méthodologique évident était de dépasser ma subjectivité de chercheur unique, la particularité de mon approche théorique et plus généralement de compenser l'orientation singulière de mon regard ethnologique par un autre qui la relativiserait

L'enquête : axes, thèmes et techniquesLa première phase de l'enquête (durée : 2 mois)

Elle était entièrement orientée vers la réalisation des objectifs intermédiaires 1, 2 et 3 dont le but était de mettre en évidence des institutions et des stratifications sociales susceptibles de révéler des différences locales dans la maitrise et dans la spécialisation des compétences en matière de gestion des terroirs.

L'inventaire

24 Atteindre ces objectifs impliquait que les enquêteurs réalisent, au préalable, un important et systématique travail d'inventaire (emploi de la technique d'observation indirecte par passage unique dans tous les quartiers) de tous les villages, communes et secteurs de communes administrativement reconnus par les autorités départementales et provinciales. Cette démarche peut sembler, de prime abord, surprenante mais sa nécessité s'est imposée à moi dès que je me suis rendu compte que personne, tant au niveau du projet, qu'à celui de l'Administration provinciale, ne savait exactement combien de "villages" relevaient de sa juridiction administrative.

L'entretien centré sur thème

Par contre, les enquêtes sur les catégories sociales, économiques et symboliques pertinentes ont été conduites à l'aide de la technique d'entretien centré sur thème qui fut utilisée avec les autorités administratives, coutumières et religieuses des différents quartiers (qui constituaient les niveaux micro-locaux de décision facilement repérables sur le terrain). Nous avions déjà remarqué, à l'occasion d'enquêtes antérieures, que selon le thème abordé par l'enquête, l'interlocuteur du moment nous renvoyait soit au délégué administratif du village (CR), soit au chef coutumier, soit encore au maitre de la terre. Manifestement, tout le monde s'accordait implicitement sur le principe selon lequel celui qui détient le pouvoir de faire a aussi le pouvoir de dire! Le passage systématique nécessaire au travail d'inventaire préalable a fortement bénéficié à cette phase de l'enquête.

Entretiens libres individuels ou en groupes

Enfin, des entretiens libres ont été conduits avec toutes sortes de personnes, individuellement ou en groupes, dont des jeunes villageois et des encadreurs agricoles.

La cartographie des conflits

La cartographie contradictoire des limites des aires de compétence respectives des différentes autorités politiques ou religieuses, nationales ou locales, a été réalisée dans cette phase afin de mettre en évidence les modalités conflictuelles ou non de leurs articulations spatiales. Les résultats de cette cartographie polémologique nous permirent d'établir, pour une zone donnée, une correspondance entre l'existence de 
conflits de pouvoirs locaux et le refus de contracter des contrats villageois puisque tous les villages ayant refusé de contracter se trouvaient localisés précisément dans ces zones-là.

La seconde phase de l'enquête (durée : 1 mois)Etudes de cas : approche intensive et qualitative

La seconde phase de l'enquête a consisté en études de cas observés au niveau micro-local. Ces observations ont été conduites à partir de trois axes dans une perspective beaucoup plus intensive et qualitative (recueil de données plus "conceptuelles") que celle de la première phase (recueil de données plus "objectives") : 1er axe

Objectif : étudier la diversité des droits d'appropriation collective et individuelle de la terre et enregistrer les catégories juridiques coutumières (entretiens dirigés individuels ou de groupe). Cette approche était particulièrement importante puisque les règles et les pratiques sociales qu'elle était susceptible de révéler se trouvaient au confluent de l'interaction des différentes logiques identifiées dans la première phase de l'étude.

\section{2ème axe}

Objectif : répondre à la question de savoir si les notions de "village" et de "terroir" ont un sens pour le paysan du Ganzourgou et si oui, lequel (technique d'entretien centré sur thème et observation). Une des approches possibles consiste à repérer la manière dont les paysans conceptualisent leurs savoirs en matière d'environnement naturel en essayant de dégager quels sont les représentations et les pratiques (économiques ou autres) qui les fondent. Les toponymies de terroirs ont constitué un des meilleurs indicateurs de ces représentations (4 toponymies ont été recueillies en des lieux différents et ont fait l'objet d'analyses typologiques).

\section{3ème axe}

Etudier quelques conflits et problèmes localisés ayant été repérés lors de la première phase de l'enquête (entretiens libres croisés et entretiens centrés). Cette approche présente l'intérêt évident d'observer in situ et à chaud l'interaction des différentes logiques d'action à l'œuvre dans les stratégies paysannes effectivement mises en œuvre. Les "blocages" paysans qui traduisent, en partie, la complexité des jeux de pouvoir s'exerçant autour du contrôle de l'espace, ne constituent pas une situation exceptionnelle. $\mathrm{Au}$ contraire, ces configurations locales où s'enchevêtrent et s'emboîtent différents types de droits sur la terre sont en l'occurrence relativement fréquentes. Elles témoignent d'une réalité paysanne dynamique qui intègre tout à la fois les dimensions symboliques propres à la culture régionale, à l'histoire locale et aux enjeux économiques actuels.

5 Le rapport final comprend 8 volumes + les cartes (500 pages au total) dont :

1 volume introductif "Généralités et synthèse" présentant la méthodologie, des études de cas de conflits et un lexique plus 7 volumes organisés en "fichiers de villages" (un volume par département).

Chaque fiche renvoyait à la description standardisée d'un village. Chaque village a fait l'objet d'une fiche plus ou moins détaillée selon les données disponibles. Il était situé, délimité, et caractérisé par ses différents quartiers, la catégorie sociale "émique" de ses habitants, le pouvoir dont il était le dépositaire, les associations villageoises s'y trouvant et les types de conflits repérés. Les fiches ont été conçues comme évolutives c'est-à-dire susceptibles d'enrichissement en fonction de toute nouvelle donnée d'ordre 
économique, agronomique, écologique, social ou historique qui aurait été recueillie par un agent du projet dans l'exercice de ses activités.

Un jeu de 6 cartes au 1/10000ème couvrant la totalité de la province du Ganzourgou et signalant les limites des diverses trames territoriales administratives, coutumières et rituelles ainsi que leurs centres d'autorité (préfectures, chefferies, maîtrises de terre, chapelles, mosquées...) a été réalisé. La cartographie permit de mettre en évidence les zones de tensions dues à des chevauchements de trames foncières ayant conduit à des conflits d'autorité entre diverses autorités locales.

Le produit final, le rapport, a été conçu comme un outil de travail qui devait être facilement compréhensible par un non anthropologue et directement accessible et utilisable quotidiennement par tous les agents du projet. Cette forme de présentation, accessible et systématique avait, je crois, l'avantage de constituer un bon outil de communication entre anthropologues et professionnels du développement qui aurait pu servir de support à un dialogue autour de "la définition des questions et des domaines respectifs et de leur articulations, qui constitue la première étape d'une approche pluridisciplinaire."(Desclaux 1992 : 305)

En guise de bouclage de procédure

Qu'advint-il donc des contrats villageois de développement? J'étais convaincu que les CVD constituaient une véritable innovation dans la manière de concevoir l'interaction entre les experts et les agents d'un projet de développement et les destinataires-paysans. La notion de "contrat villageois" évoquait chez moi la représentation d'un rapport égalitaire et librement consenti entre des "partenaires". Le rapport avait donc été explicitement conçu dans le but de transmettre les résultats anthropologiques sous une forme compréhensible pour les non-anthropologues et, en particulier, de fournir aux agents du Projet un outil de connaissance sociale et économique générale de chaque milieu villageois particulier permettant toutefois, dans chaque cas, d'identifier les "décideurs" locaux, ceux qui détenaient réellement ${ }^{14}$ un pouvoir de décision ou d'influence à la décision. La considération sous-jacente à ce choix était que la situation contractuelle exigeait pour chaque contractant de connaître, parmi ses interlocuteurs, quels étaient les vrais décideurs ainsi que la nature et les limites de leurs pouvoirs spécifiques.

41 Mais pour qu'une telle innovation réussisse, toutes les interactions sociales qui ne présentaient pas ces caractères devaient changer. Parmi ces pratiques, la manière habituelle que les cadres du projet avaient de se rendre dans les villages fournit un bon exemple : le style "commandant qui va haranguer les villageois réunis sur la place du village" n'était plus compatible avec la notion de Contrat Villageois.

Il aurait fallu dorénavant procéder autrement en entamant des négociations sérieuses avec les "vrais" décideurs locaux sur le contenu économique du Contrat et sur les devoirs et obligations réciproques de partenaires qui se doivent un respect mutuel. La Direction du projet aurait dû abandonner la langue de bois, reconnaître les intérêts et les savoirs locaux, se départir de sa toute puissance et négocier des compromis... C'était trop demander à des cadres et des experts (aussi bien expatriés que nationaux) dont l'autorité sur les paysans, souvent empreinte d'arrogance, s'était toujours appuyée sur la technicité indiscutable de leur savoir spécialisé.

43 Négocier c'était, pour certains esprits, commencer à mettre en question la supériorité de ce savoir qui justifiait la position occupée dans l'organigramme du projet. Pour ceux -là ce n'était pas la réussite ou l'échec des objectifs déclarés du projet qui constituait le 
véritable enjeu du pouvoir, mais la conservation (ou l'amélioration) de la position occupée, considérée et valorisée avant tout comme la source de ressources de toutes sortes, qui présentent l'intérêt majeur d'être négociables ailleurs, dans les autres réseaux sociaux, économiques ou politiques auxquels on appartient ou souhaite appartenir.

Finalement, ce n'est pas tant la "lettre" des contrats de développement villageois mais plutôt leur "esprit" qui importe vraiment. Un esprit fondé sur l'état de partenariat, lui-même produit par un processus permanent de négociation et de coopération. Les conditions de mise en place de l'EAR et celles d'utilisation de ses résultats ont montré que cette conception du partenariat, encore loin d'être partagée, demeurait un idéal à atteindre à l'horizon du développement.

\section{BIBLIOGRAPHIE}

Bierschenk Thomas \& Olivier de Sardan Jean-Pierre (1994) "ECRIS : Enquête collective rapide d'identification des conflits et des groupes stratégiques", Bulletin de l'APAD, n 7 :35-43.

Bouju Jacky (1990) "Pratiques économiques et structures sociales : exemples dogon au Mali" in D. Fassin et Y. Jaffré (coordinateurs), Sociétés, santé et développement, coll. "Médecine tropicale", Universités Francophones, Ellipses / Aupelf : 155-166.

Bouju Jacky (1991) "Pouvoirs et légitimités sur le contrôle de l'Espace rural. L'exemple du Ganzourgou (Burkina Faso)", in J. P. Olivier de Sardan et E. Paquot (sous la direction de) D'un savoir à l'autre, Les agents de développement comme médiateurs, G.R.E.T., Ministère de la Coopération et du Développement : 60-70.

Bouju Jacky \& Brand Roger (1989), Analyse socio-anthropologique des trames foncières dans la province du Ganzourgou, Aménagement des Vallées des Voltas, Projet U.P.1-Zorgo, T.1 \& 2, 8 fasc., 12 cartes : 520 p. multigr.

Desclaux Alice (1992) "Le 'RAP' et les méthodes anthropologiques rapides en santé publique", Cahiers d'études et de recherches francophones. Santé, vol. 2 (5), sept-oct. : 300-6.

Lefèvre Pierre \& Kolsteren Patrick - 1994 "Le développement comme arène : implications pour l'évaluation des projets" Bulletin de l'APAD, nº 8 - décembre 1994 : 19-30.

Olivier de Sardan Jean-Pierre (1989) "L'anthropologie du changement social et du développement comme ambition théorique", Bulletin de l'APAD, n¹- juin 1989 : 7.

Olivier de Sardan Jean-Pierre (1990) "Sociétés et développement" in Sociétés, santé et développement, Didier Fassin et Yannick Jaffré (coord.), Coll. "Médecine tropicale", Universités Francophones, Ellipses / Aupelf : 28-37.

Vidal Laurent (1994) "L'anthropologie, la recherche et l'intervention sur le Sida : enjeux méthodologiques d'une rencontre" Bulletin de l'APAD, nº 8 - décembre 1994 : 79-91. 


\section{NOTES}

1.Cf A. Desclaux, 1992 : 300. L'EAR que je présente ici prend la forme d'un "guide d'analyse spécifique", il ne s'agit pas d'un "guide rapide" et encore moins d'un "guide méthodologique général". Ma première expérience d'expertise et de collaboration pluridisciplinaire dans le champ du développement rural remonte à 1980. Celle qui sert ici de référence a pris place en 1987-88 alors que j'étais depuis six ans enseignant-chercheur en ethnologie à l'Université de Ouagadougou (Burkina Faso). 2.Il serait intéressant de disposer d'une évaluation globale des projets (UP 1 - Zorgho en 1988 et PVNY en 1989) qui ont commandé les études réalisées par cette méthode. Cf. Lefèvre P. \& Kolsteren P. 1994.

3.Surtout dans le domaine de la santé publique où la collaboration est, semble-t-il, plus régulière et incontournable qu'elle ne semble l'être dans celui du développement urbain ou économique rural. Voir à cet égard : Scrimshaw S.C.M., Hurtado E. Rapid Assessment Procedures for Nutrition and Primary Health Care: Anthropological approaches to improving programme effectiveness, Los Angeles : UCLA Latin American Center Publications, University of California, 1987 ; Manderson L., Aaby P., "Can rapid anthropological procedures be applied to tropical deseases ?" Health Pol. Plan. 1992, $\mathrm{n}^{\circ} 7$ : 46-55 ; Scrimshaw S.CM., Carballo M., Ramos L., Blair B.A., "The AIDS Rapid Anthropological Assessment Procedures : A tool for health education planning and evaluation" Health Education Q. 1991, n 18 : 111-23 ; Hurtado E., "Use of rapid anthropological procedures by health personnel in Central America" Food Nutritional Bulletin 1990, n¹2 : 310-2 ; Gruénais M.-E., "Appliquer l'anthropologie au domaine de la santé. Entre recherche et expertise", Bulletin de l'APAD, nº, décembre 1994 :52-59 ; Vidal Laurent (1994).

4.Pour des raisons de nature politique auxquelles se heurte assez souvent la démarche anthropologique. Il faut se souvenir qu'à l'époque le gouvernement révolutionnaire du président Thomas Sankara avait mis en place les Comités de Défense de la Révolution (CDR) qui constituaient la structure de base de la nouvelle administration territoriale (qui comprenait, en ordre croissant de hiérarchie : les CDR de secteurs de commune, de commune ou de village, de préfecture, de province, etc.). Pour le pouvoir révolutionnaire, la structure des CDR était supposée être confrontée, en tout lieu, aux forces conservatrices et réactionnaires censées être incarnées, en milieu rural, par les chefferies coutumières et les autres autorités pré-révolutionnaires. En tout état de cause, ma proposition d'identifier et de localiser les centres de décisions locaux afin de pouvoir répondre à la demande présentée par le projet a suscité, en 1987, la plus grande méfiance de la part des responsables du projet qui n'y ont donné suite que l'année suivante, en 1988, après que des changements politiques importants soient intervenus au niveau national.

5.Le mot "socio-anthropologie" était moins inquiétant pour les bailleurs de fonds et les cadres-techniciens du développement que "anthropologie" qui, lui-même, passait mieux que "ethnologie"!

6.Cf. Bouju, $1990: 165$.

7.Les illusions entretenues par les tiers-mondistes européens sur "l'authenticité de la vie au village" semblent partagées, en partie, dans un certain milieu urbain et scolarisé ouest-africain où surgit, de manière récurrente (et en parallèle avec d'autres imaginaires), un ensemble de représentations idéales du village parmi lesquelles on 
trouve toujours la nostalgie d'un univers consensuel à la fois lieu de coopération, de partage et d'expression d'une solidarité sans faille (Idem Bouju, $1990: 165$ ).

8.Des observations identiques ont été effectuées au Mali, voir (Bouju, 1990 : 156).

9.C'est-à-dire le concept de "village" ou celui de "terroir" tels qu'ils sont définis dans les manuels de géographie des pays développés.

10.Dont on feint d'ignorer les intérêts.

11.J'ai compris, beaucoup plus tard, que la Direction burkinabè du projet n'était pas convaincue de la nécessité de réaliser une étude anthropologique sur les trames foncières. En fait, elle ne s'y était résolue, avec une absence totale d'enthousiasme, que parce que les bailleurs de fonds (et en particulier la Caisse Centrale de Coopération qui était le principal financeur) avaient subordonné la reconduction du financement du projet à la réalisation de cette étude dont ils attendaient des "clés" pour débloquer la "négociation" sur les Contrats Villageois de Développement.

12.Certaines légitimités foncières parfaitement légales ne sont pas reconnues par les villageois du Ganzourgou (ou bien considérées comme secondaires) tandis que d'autres, endogènes au milieu, historiquement plus anciennes et qu'on pourrait qualifier de "traditionnelles", sont universellement reconnues par le monde paysan et, surtout, sont considérées comme prééminentes. Ces dernières, bien que n'étant plus légales du point de vue juridique de l'Etat révolutionnaire burkinabè, restent profondément déterminantes des logiques d'action et de réaction paysannes.

13.Sur la valeur heuristique du conflit dans l'appréhension des problèmes de développement voir Bierschenk,T. \& Olivier de Sardan, J.-P., 1994 : 36-37.

14.Ces décideurs réels étaient bien souvent différents des autorités officielles !

\section{AUTEUR}

JACKY BOUJU

Université de Provence Aix-Marseille 1, SHADYC EHESS-Marseille. 